\title{
POWER BOUNDED STRICTLY CYCLIC OPERATORS
}

\author{
ERIK J. ROSENTHAL
}

\begin{abstract}
We show that a power bounded hereditarily strictly cyclic operator on Hilbert space is similar to a contraction. We also show that certain "almost unitary" operators are not strictly cyclic.
\end{abstract}

Recall that the operator $T$ is power bounded if there exists a positive real number $M$ such that $\left\|T^{n}\right\| \leqslant M$ for $n=1,2,3, \ldots$ Sz.-Nagy [16] proved that every compact power bounded operator is similar to a contraction and asked whether the hypothesis of compactness can be removed. Foguel [3] (see also Halmos [5]) showed that it cannot be removed-there is a power bounded operator that is not similar to a contraction. (It is still not known if every polynomially bounded operator is similar to a contraction. See Ghatage [4] for some related results.)

Given an operator $T$, let $\mathcal{Q}(T)$ denote the uniformly closed algebra generated by $T$ and the identity. Lambert [11] defined $T$ to be strictly cyclic if there exists a vector $x_{0}$ such that $\left\{A x_{0}: A \in \mathbb{Q}(T)\right\}$ is the entire space. $T$ is hereditarily strictly cyclic if its restriction to every invariant subspace is strictly cyclic. There are a number of known results about strictly cyclic operators-see [6], [7], [8], [10], [11], [12], [13], [14].

In this paper we prove that a power bounded hereditarily strictly cyclic operator is similar to a contraction. We require three lemmas.

LEMMA 1. If $T$ is a strictly cyclic operator, and if $\Re$ is an invariant subspace of $T$, then the compression of $T$ to $\mathfrak{M}^{\perp}$ is strictly cyclic.

Proof. Let $P$ be the projection onto $\mathfrak{T}^{\perp}$, and let $e$ be a strictly cyclic vector for $T$; i.e.,

$$
\{A e: A \in \mathbb{Q}(T)\}=\mathcal{H} \text {. }
$$

Let $f=P e$ and $m=e-f$. Then it suffices to show that

$$
\{\text { PAf: } A \in \mathbb{Q}(T)\}=\Re^{\perp} \text {. }
$$

Note that $P A m=0$ for every $A$ in $Q(T)$. Now, given $x \in \Re^{\perp}$, choose $A$ in $Q(T)$ such that $x=A e$. Then

$$
P A f=P A(f+m)=P A e=P x=x,
$$

and the proof is complete.

LEMMA 2. If $T$ is hereditarily strictly cyclic and power bounded, and if 
$\sigma(T)=\left\{\lambda_{0}\right\}$, where $\left|\lambda_{0}\right|=1$, then $T$ acts on a one-dimensional space.

Proof. By replacing $T$ by $\frac{1}{\lambda_{0}} T$, we may assume that $\lambda_{0}=1$. Lambert [11] proved that $T$ strictly cyclic implies that every point in the spectrum of $T$ is compression spectrum. Thus, 1 is an eigenvalue for $T^{*}$. So choose a unit vector $e$ such that $T^{*} e=e$, and let

$$
T=\left(\begin{array}{cc}
1 & 0 \\
A & B
\end{array}\right)
$$

be the decomposition of $T$ with respect to the decomposition of $\mathcal{H}=\bigvee\{e\}$ $\oplus\{e\}^{\perp}$. We claim that $\{e\}^{\perp}=\{0\}$. If not, since $T$ is hereditarily strictly cyclic and $B=T \mid\{e\}^{\perp}, B$ is strictly cyclic. Also, $\sigma(B)=\{1\}$, so there is a unit vector $f \perp e$ with $B^{*} f=f$. Then decompose $T$ with respect to the decomposition $\mathcal{H}=\bigvee\{e, f\} \oplus\{e, f\}^{\perp}$ :

$$
T=\left(\begin{array}{lll}
1 & 0 & 0 \\
\lambda & 1 & \\
C & D
\end{array}\right)
$$

Since $T$ is power bounded, $\left(\begin{array}{ll}1 & 0 \\ 1 & 1\end{array}\right)$ is power bounded. Since $\left(\begin{array}{ll}1 & 9 \\ 1 & 1\end{array}\right)^{n}=\left(\begin{array}{ll}1 & 9 \\ n \lambda & 9\end{array}\right), \lambda$ must be 0 . Since $\{e, f\}^{\perp} \in$ Lat $T,\left(\begin{array}{ll}1 & 9 \\ 0 & 1\end{array}\right)$ is strictly cyclic by Lemma 1 . But the identity is strictly cyclic only on a one-dimensional space, so $\{e\}^{\perp}$ must be $\{0\}$.

We should perhaps note that the above lemma implies the following well known fact.

THEOREM 1 (SZ.-NAGY). A power bounded operator acting on a finite dimensional space is similar to a contraction.

Proof. By the Jordan canonical form theorem, it suffices to prove the theorem for a single Jordan block, so consider an operator

$$
T=\left(\begin{array}{cccc}
\gamma & 1 & & 0 \\
& \gamma & 1 & \\
0 & \cdot & \cdot & \cdot \\
& & \ddots \gamma & 1
\end{array}\right)
$$

Since $T$ is power bounded and $\left\|T^{n}\right\| \geqslant|\gamma|^{n},|\gamma| \leqslant 1$. If $|\gamma|=1$, then Lemma 2 implies that $T$ acts on a one-dimensional space, and we are done. If $|\gamma|<1$, then $T$ is similar to

$$
S=\left(\begin{array}{ccccc}
\gamma & (1-|\gamma|) & & & \\
& \gamma & (1-|\gamma|) & \\
& & \cdot & \ddots & \\
& 0 & & \cdot(1-|\gamma|) \\
\gamma & & & & \gamma
\end{array}\right)
$$


(by the Jordan canonical form theorem). Then $\|S\| \leqslant\|\gamma I\|+\left\|(1-|\gamma|) S_{0}\right\|$ where $S_{0}$ is a shift all of whose weights are 1 . Thus $\|S\| \leqslant|\gamma|+1-|\gamma|=1$.

The next lemma is a slight variant of Theorem 4 of Barnes [1] (which is the case where $\|T\|=1$ ).

LEMMA 3. If $T$ is strictly cyclic and power bounded, and if $\lambda \in \sigma(T),|\lambda|=1$, then $\lambda$ is an isolated point of $\sigma(T)$.

Proof. Suppose that $\left\|T^{n}\right\| \leqslant M$ for all $n$, and that $\lambda$ is as stated. By replacing $T$ by $\frac{1}{\lambda} T$, we may assume that $\lambda=1$. Let $S=\frac{1}{2}(T+1)$. Then for each $n$,

$$
\left\|S^{n}\right\|=\frac{1}{2^{n}}\left\|(T+1)^{n}\right\| \leqslant \frac{1}{2^{n}} \cdot M(1+1)^{n}=M .
$$

Since $\left\{S^{n}\right\}$ is bounded, some subsequence $\left\{S^{n_{i}}\right\}$ converges weakly to some operator $R$.

The algebra $\mathscr{Q}(T)$ is maximal abelian by a result of Lambert [10]. The spectrum of each element of $\mathscr{Q}(T)$ is the same as its spectrum relative to the Banach algebra $\mathcal{Q}(T)$ (cf. [15, p. 4]). Thus, if $\mathfrak{N}$ is the set of nonzero complex homomorphisms of $\mathscr{Q}(T)$, then

$$
\sigma(T-R)=\{\phi(T-R): \phi \in \mathfrak{T}\} .
$$

Now, if $\phi(T) \neq 1,|\phi(T)+1|<2$ since $|\phi(T)| \leqslant 1$. Hence $|\phi(S)|<1$, and $\phi\left(S^{n}\right) \rightarrow 0$. Lambert [11, Theorem 1.7] proved that every linear functional on $\mathcal{Q}(T)$ is weakly continuous. So $\phi(T) \neq 1$ implies $\phi(R)=0$, and $\phi(T)=1$ implies $\phi(R)=1$ for every $\phi \in \mathfrak{N}$. Hence

$$
\sigma(T-R)=\{\phi(T)-\phi(R): \phi \in \mathfrak{N}\}=(\{\phi(T): \phi \in \mathfrak{N}\} \backslash\{1\}) \cup\{0\} .
$$

Since $\sigma(T-R)$ must be closed, 1 must be an isolated point of $\sigma(T)$; (if $\left\{\lambda_{n}\right\} \rightarrow 1$ and $\left\{\lambda_{n}\right\} \subset \sigma(T) \backslash\{1\}$, then $\left\{\lambda_{n}\right\} \subset \sigma(T-R)$, and since $1 \notin$ $\sigma(T-R), \sigma(T-R)$ would not be closed).

THEOREM 2. A power bounded hereditarily strictly cyclic operator is similar to a contraction.

Proof. Let $T$ be power bounded and hereditarily strictly cyclic. The spectral radius of $T$ is at most 1 ; by the preceding lemma, $\sigma(T) \cap\{z$ : $|z|=1\}$ is some finite set $\left\{\lambda_{1}, \ldots, \lambda_{n}\right\}$. (If $\sigma(T) \cap\{z:|z|=1\}=\varnothing$, the first part of the proof is not required.) For each $j$ let

$$
P_{j}=\frac{1}{2 \pi i} \int_{\Gamma_{j}}(z-T)^{-1} d z
$$

where $\Gamma_{j}$ is a circle about $\lambda_{j}$ such that $\Gamma_{j} \cap \sigma(T)=\varnothing$ and the interior of $\Gamma_{j}$ meets $\sigma(T)$ only in $\left\{\lambda_{j}\right\}$. Then each $P_{j}$ is a Riesz projection of $T$ (cf. [15, Chapter 2]). If $\Re_{j}=\Re\left(P_{j}\right)$, then the well-known theorem of Riesz (cf. [15, p. 31]) implies that $\Re_{j}$ is invariant under $T$, and that $\sigma\left(T \mid \Re_{j}\right)=\left\{\lambda_{j}\right\}$ for each $j$. Clearly $T \mid \Re_{j}$ is also power bounded, so $\Re_{j}$ is one-dimensional by Lemma 
2. Now if $\Re_{4}=\bigvee_{j=1}^{n} \Re_{j}$, then $\Re$ is a finite-dimensional invariant subspace of $T$, so $T \mid \mathfrak{T}$ is similar to a contraction by Theorem 1. Also, $\Re$ has an invariant complement $\Re$, $\left(\Re\right.$ is the null space of $\left.P_{1}+P_{2}+\ldots+P_{n}\right)$. Evidently $\sigma(T \mid \Re) \subset\{z:|z|<1\}$. Thus, by Rota's theorem (cf. [15, Theorem 3.28]) $T \mid \Re$ is similar to a part of the backwards shift of multiplicity the dimension of $\Re$. Obviously every such part is a contraction.

Now, $\mathcal{K}=\Re \oplus \Re$, and $T \mid \Re$ and $T \mid \Re$ are each similar to a contraction. Since $T$ is similar to $(T \mid \mathscr{\Re}) \oplus(T \mid \mathcal{X})$, it follows that $T$ is similar to a contraction.

The next corollary is immediate. It contains the fact that power bounded operators on finite-dimensional spaces are similar to contractions by the Jordan canonical form theorem.

COROLlary. A power bounded operator which is the (not necessarily orthogonal) direct sum of a finite number of hereditarily strictly cyclic operators is similar to a contraction.

Barnes [1, Theorem 8] proves that no hyponormal operator is strictly cyclic on an infinite-dimensional space. In particular, there are no strictly cyclic unitary operators on infinite-dimensional spaces. The next theorem is a mild generalization of this fact about unitary operators.

THEOREM 3. If $T$ is strictly cyclic, power bounded, and invertible, and if there is $a k \geqslant 1$ such that

$$
\lim _{n \rightarrow \infty} \frac{1}{n^{k}}\left\|T^{-n}\right\|=0
$$

then $T$ acts on a finite-dimensional space.

Proof. Since $T$ is power bounded, $\sigma(T) \subset\{z:|z| \leqslant 1\}$. Choose $M>0$ such that $\left\|T^{-n}\right\| \leqslant M \cdot n^{k}$. Then the spectral radius of $T^{-1}$ is at most $\lim _{k \rightarrow \infty} M^{1 / n}\left(n^{k}\right)^{1 / n}=1$, so $\sigma\left(T^{-1}\right) \subset\{z:|z| \leqslant 1\}$. Since $\sigma\left(T^{-1}\right)=\{1 / z$ : $z \in \sigma(T)\}$, it follows that $\sigma(T) \subset\{z:|z|=1\}$. Now Lemma 3 implies that $\sigma(T)=\left\{\lambda_{1}, \lambda_{2}, \ldots, \lambda_{n}\right\}$ for complex numbers $\lambda_{i}$ of modulus 1 . As in the proof of Theorem 2, let $\mathscr{N}_{i}$ be the Riesz subspace such that $\sigma\left(T \mid \mathscr{N}_{i}\right)=\left\{\lambda_{i}\right\}$. Since each $\mathfrak{T}_{i}$ has an invariant complement, $\mathfrak{T}_{i}$ is invariant under $T^{-1}$ for each $i$. Thus,

$$
\lim _{n \rightarrow \infty} \frac{1}{n^{k}}\left\|\left(T \mid \Re_{i}\right)^{n}\right\|=\lim _{n \rightarrow \infty} \frac{1}{n^{k}}\left\|\left(T \mid \Re_{i}\right)^{-n}\right\|=0 .
$$

These growth conditions together with the fact that $\sigma\left(T \mid \Re_{i}\right)$ is the singleton $\left\{\lambda_{i}\right\}$ imply that $\left(T \mid \mathscr{N}_{i}-\lambda_{i}\right)^{k}=0$ by a theorem of Hille (see [9. p. 128]). This implies $T$ is an algebraic operator, so each of its cyclic invariant subspaces is finite-dimensional. Since $T$ is cyclic, it acts on a finite-dimensional space.

\section{REFERENCES}

1. B. Barnes, Operators with a strictly cyclic vector, Proc. Amer. Math. Soc. 41 (1973), 480-486. 
2. M. Embry, Maximal invariant subspaces of strictly cyclic operator algebras, Pacific J. Math. 49 (1973), 45-50.

3. S. R. Foguel, A counterexample to a problem of Sz.-Nagy, Proc. Amer. Math. Soc. 15 (1964), 788-790.

4. P. Ghatage, Generalized algebraic operators, Proc. Amer. Math. Soc. 52 (1975), 232-236.

5. R. P. Halmos, On Foguel's answer to Sz.-Nagy's question, Proc. Amer. Math. Soc. 15 (1964), 791-793.

6. J. H. Hedlund, Strongly strictly cyclic weighted shifts, Proc. Amer. Math. Soc. 57 (1976), 119-121.

7. D. A. Herrero, Transitive operator algebras containing a subalgebra of finite strict multiplicity, Rev. Un. Mat. Argentina 26 (1972), 77-83.

8. Operator algebras of finite strict multiplicity, Indiana Math. J. 22 (1972), 13-24.

9. E. Hille and R. S. Phillips, Functional analysis and semi-groups, Amer. Math. Soc. Colloq. Publ., vol. 31, Amer. Math. Soc., Providence, R. I., 1957.

10. E. Kerlin and A. L. Lambert, Strictly cyclic shifts on lp, Acta Sci. Math. (Szeged) 35 (1973), 87-94.

11. A. L. Lambert, Strictly cyclic operator algebras, Dissertation, Univ. of Michigan, 1970.

12. __ Strictly cyclic weighted shifts, Proc. Amer. Math. Soc. 29 (1971), 331-336.

13. Strictly cyclic operator algebras, Pacific J. Math. 39 (1971), 717-726.

14. A. L. Lambert and T. R. Turner, The double commutant of invertibility weighted shifts, Duke Math. J. 39 (1972), 385-389.

15. H. Radjavi and P. M. Rosenthal, Invariant subspaces, Springer-Verlag, Berlin and New York, 1973.

16. B. Sz.-Nagy, Completely continuous operators with uniformly bounded iterates, Publ. Math. Debrecen 4 (1959), 82-92.

Department of Mathematics, State University of New York, College at New Paltz, NeW PALTZ, New YoRK 12562 